\title{
Reação de híbridos de milho e comparação de métodos para avaliação da Cercosporiose e Mancha Branca
}

\author{
André H. de Brito, Renzo G. von Pinho, Álvaro de O. Santos \& Sidnei dos Santos \\ Departamento de Agricultura, Universidade Federal de Lavras, 37200-000, Lavras, MG, Brasil
}

Autor para correspondência: Renzo G. von Pinho, e-mail: renzo@ufla.br

\section{RESUMO}

O objetivo desse trabalho foi avaliar o nível de resistência de 12 híbridos comerciais de milho à cercosporiose, causada por Cercospora zeae-maydis, e à Mancha Branca, causada por Pantoea ananatis e comparar a eficiência das estimativas da área abaixo da curva de progresso das doenças e da estabilidade fenotípica, na avaliação da resistência a esses patógenos. Foram conduzidos três experimentos, no ano agrícola de 2007/2008, em três locais. Utilizou-se o delineamento em blocos ao acaso com três repetições. Foram realizadas cinco avaliações da severidade das doenças, em intervalos de sete dias, a partir dos 80 dias da emergência, por meio de uma escala de notas variando de 1 (altamente resistente) a 9 (altamente suscetível). Foram estimadas a área abaixo da curva de progresso da doença (AACPD) e o coeficiente de regressão linear $\left(\mathrm{b}_{1}\right)$ entre a variável independente época de avaliação $(\mathrm{x})$ e a variável dependente severidade da doença $(y)$ e o coeficiente de determinação $\left(\mathrm{R}^{2}\right)$. Observou-se que, ambas as metodologias utilizadas mostraram-se eficientes na discriminação do nível de resistência dos híbridos avaliados à Cercosporiose e à Mancha Branca, permitindo a classificação de modo semelhante. Os híbridos mais resistentes às duas doenças foram o AG7088, AG7010 e 2B707 e os mais suscetíveis o 30F44, 30F53 e AG8021. Os híbridos 30K64, DKB177, DKB390 e Impacto apresentaram comportamento variável em função dos locais de avaliação.

Palavras-chave: Cercospora zeae-maydis, Pantoea ananatis, estabilidade fenotípica, doenças fúngicas.

\begin{abstract}
Reaction of commercial corn hybrids and comparison of methods for evaluation of maize White Spot and Gray Leaf Spot

With the objective of evaluating the resistance level of commercial corn hybrids and comparing the efficiency of the estimates of the area under the disease progress curve (AUDPC) and that of the phenotypical stability parameters in the evaluation of resistance maize white spot and gray leaf spot, three experiments were carried out in the agricultural year of 2007/2008 in three environments. Twelve commercial maize hybrids were used, in a randomized block experimental design with three replications. Five evaluations of disease severity (maize white spot and gray leaf spot) based on visual symptoms were performed at seven-day intervals from the $80^{\text {th }}$ day after maize emergence, ranging from 1 (highly resistant) to 9 (highly susceptible). The area under the disease progress curve (AUDPC) was estimated, as were the phenotypical stability parameters, i.e., the linear regression coefficient $\left(b_{b}\right)$ between the independent variable evaluation time $(\mathrm{x})$, and the dependent variable, disease severity $(\mathrm{y})$ and the determination coefficient $\left(\mathrm{R}^{2}\right)$. It was found that in the case of maize white spot and maize gray leaf spot, both methodologies used proved to be effective in the discrimination of the resistance level of the hybrids, enabling them to be ranked in a similar way. The most resistant hybrids to maize white spot and maize gray leaf spot were AG7088, AG 7010 and 2B707, and the most susceptible were 30F44, 30F53 and AG8021. The 30K64, DKB177, DKB390 and Impacto hybrids showed variable levels of resistance with the environment.
\end{abstract}

Key words: Cercospora zeae-maydis, Pantoea ananatis, fungal disease, phenotypical stability.

\section{INTRODUÇ̃̃O}

A cultura do milho no Brasil, pela sua ampla distribuição geográfica, ocupando as mais diversas condições edafoclimáticas, está sujeita ao ataque de um grande número de doenças, muitas das quais podem causar perdas significativas à cultura (Pozar et al., 2009). Estas perdas, associadas principalmente às doenças foliares, têm causado ampla discussão sobre estratégias de manejo que visem o seu controle de forma sustentável. A partir do

Parte da Tese de Doutorado do primeiro autor. Universidade Federal de Lavras. Lavras MG. 2010. ano 2000, a Cercosporiose do milho, causada pelo fungo Cercospora zea-maydis, começou a assumir proporções epidêmicas em várias regiões do país (Juliatti et al., 2004). Essa doença foi responsável pela substituição de vários híbridos comerciais suscetíveis que, apesar de seu alto potencial produtivo, chegaram a reduções na produtividade de grãos de até $40 \%$ (Brito et al., 2007). Hoje, a incorporação de resistência genética a Cercospora zea-maydis está entre os principais objetivos dos programas de desenvolvimento de híbridos para as regiões onde essa doença é prevalente. Outra doença foliar, de grande importância no Brasil, é a Mancha Branca, causada pela bactéria Pantoea ananatis (PaccolaMeirelles et al., 2001). Segundo Fernandes \& Oliveira 
(1997), os híbridos suscetíveis à Mancha Branca podem chegar a apresentar perdas em torno de $60 \%$ na produção.

Sabe-se que a utilização de híbridos resistentes a essas doenças é o método mais eficiente, racional e econômico para evitar ou diminuir os danos por elas causados. Como os programas de melhoramento de milho são muito dinâmicos, produzindo um grande número de novos híbridos anualmente, é necessário avaliar o comportamento desses materiais em relação a essas doenças, não só para direcionar futuros trabalhos de melhoramento visando à obtenção de híbridos resistentes, como para verificar o comportamento desses patógenos frente a diferentes ambientes. $\mathrm{Na}$ obtenção de híbridos resistentes, a essas doenças, existem alguns problemas que deverão ser superados. Entre estes, está o desenvolvimento de uma metodologia de avaliação que seja comparável e eficiente, porém, de fácil aplicação, considerando que alguns milhares de genótipos deverão ser avaliados (Pinho et al., 2001).

A resistência horizontal tem sido mais eficiente no controle das principais doenças foliares do milho, por atuar na maioria dos indivíduos da população dos patógenos. A eficiência da resistência horizontal está em sua suposta maior estabilidade por atuar uniformemente contra todas as raças da população de um patógeno e em sua maior durabilidade por estar menos sujeita a flutuações na população do patógeno (Pinho et al., 2001). Entre as maneiras de se identificar materiais com resistência horizontal, está a avaliação da área abaixo da curva de progresso da doença (Shanner \& Finney, 1977). Outra forma de se identificar materiais com resistência horizontal é a comparação de curvas de progresso de doenças que permitem obter informações sobre a taxa de progresso da doença durante o desenvolvimento da cultura. Neste caso, metodologias para a quantificação da estabilidade fenotípica, comumente empregadas para a avaliação de outras características agronômicas, podem ser utilizadas com sucesso (Pereira et al., 1996; Pinho et al., 2001).

Considerando a importância de se obter informações sobre o nível de resistência dos principais híbridos de milho, disponíveis na região sudeste, à Cercosporiose e à Mancha Branca, bem como a estabilidade desta resistência em relação ao progresso e comportamento destas doenças frente a diferentes ambientes, foi realizada esta pesquisa. O objetivo foi avaliar o nível de resistência de híbridos comerciais de milho à cercosporiose e à Mancha Branca e comparar a eficiência das estimativas da área abaixo da curva de progresso das doenças e da estabilidade fenotípica, na avaliação de híbridos a essas duas doenças foliares.

\section{MATERIAL E MÉTODOS}

Os experimentos foram conduzidos em três locais, na safra agrícola 2007/2008, na área experimental do Departamento de Agricultura, da Universidade Federal de Lavras, em Lavras, MG; na fazenda experimental da Fundação de Ensino Superior de Passos (FESP/UEMG), localizada em Passos MG e na área experimental do Centro Universitário de Patos de Minas (UNIPAM), em Patos de Minas MG. Em todos os locais, a condução dos experimentos foi em período de ocorrência de temperaturas, intensidade e distribuição de chuvas favoráveis ao cultivo do milho e ao progresso da Cercosporiose e da Mancha branca. Nos três locais os experimentos foram conduzidos em sistema de plantio convencional, com arações e gradagens, em áreas onde se pratica a sucessão de culturas, com o plantio de milho após milho, resultando em grande fonte de inóculo de fungos causadores de doenças foliares.

Foram avaliados doze híbridos simples com diferentes características provenientes de diferentes empresas sementeiras do Brasil (Tabela 1). Os híbridos foram selecionados de acordo com informações de suas respectivas empresas detentoras quanto ao nível de resistência à Mancha Branca à Cercosporiose e a sua utilização pelos agricultores nos locais de condução dos experimentos. O delineamento experimental utilizado foi o de blocos ao acaso, com três repetições. As parcelas experimentais foram constituídas de quatro fileiras de 5,0 $\mathrm{m}$ de comprimento, sendo as duas fileiras centrais consideradas como úteis. $\mathrm{O}$ espaçamento entre fileiras foi de $0,8 \mathrm{~m}$ e a densidade de cinco plantas por metro linear, após o desbaste.

Foi instalado um experimento em cada local, sendo a semeadura realizada na primeira quinzena de dezembro, em todos os locais. Na semeadura, foram utilizados $450 \mathrm{~kg}$ ha $^{-1}$ da fórmula $8(\mathrm{~N}): 28\left(\mathrm{P}_{2} \mathrm{O}_{5}\right): 16\left(\mathrm{~K}_{2} \mathrm{O}\right)$. Em cobertura, foram aplicados $300 \mathrm{~kg} \mathrm{ha}^{-1}$ da fórmula $30(\mathrm{~N}): 00\left(\mathrm{P}_{2} \mathrm{O}_{5}\right)$ : $20\left(\mathrm{~K}_{2} \mathrm{O}\right)$, no estádio de 4-5 folhas totalmente expandidas. Foram utilizados os mesmos tratos culturais para todos os experimentos, nos três locais. Os experimentos foram conduzidos sob condições de infecção natural. Para aumentar o potencial de inóculo das doenças em torno das áreas experimentais, foram plantadas linhas de bordadura com o híbrido AG9020, suscetível às duas doenças.

TABELA 1 - Características dos híbridos avaliados

\begin{tabular}{lcc}
\hline \hline Híbridos & Mancha-Branca* & Cercosporiose* \\
\hline DKB 390 & $\mathrm{R}$ & $\mathrm{R}$ \\
DKB 177 & $\mathrm{R}$ & $\mathrm{R}$ \\
AG 7010 & $\mathrm{R}$ & $\mathrm{R}$ \\
AG 7088 & $\mathrm{R}$ & $\mathrm{R}$ \\
AG 8088 & $\mathrm{R}$ & $\mathrm{MR}$ \\
IMPACTO & $\mathrm{MR}$ & $\mathrm{MR}$ \\
2B707 & $\mathrm{MR}$ & $\mathrm{MR}$ \\
2B587 & $\mathrm{MR}$ & $\mathrm{MR}$ \\
P30F53 & $\mathrm{MS}$ & $\mathrm{MS}$ \\
P30F44 & $\mathrm{S}$ & $\mathrm{S}$ \\
P30K64 & $\mathrm{S}$ & $\mathrm{MS}$ \\
AG 8021 & $\mathrm{MR}$ & $\mathrm{S}$ \\
\hline
\end{tabular}

*Resistência às doenças Mancha-Branca (Pantoea ananatis) e Cercosporiose (Cercospora zeae-maydis), sendo $\mathrm{S}=$ suscetível, MR $=$ moderadamente resistente, $\mathrm{MS}=$ moderadamente suscetível $\mathrm{e} \mathrm{R}=$ resistente, de acordo com informações das empresas produtoras de sementes.

Tropical Plant Pathology 36 (1) January - February 2011 
Para avaliar as doenças, foram utilizados os dados de severidade na parcela (notas), considerando a parcela como um todo, obtidos com o auxílio da escala diagramática apresentada por Pinho et al. (2001). As notas de severidade de cada doença nesta escala variam de 1 a 9 de acordo com a \% de área foliar afetada, em que: $1(0 \%), 2(1 \%), 3(>1 \%$ e $\leq 10 \%), 4(>10 \%$ e $\leq 20 \%), 5$ (>20\% e $\leq 30 \%), 6(>30 \%$ e $\leq 40 \%), 7$ ( $>40 \%$ e $\leq 60 \%), 8(>60 \%$ e $\leq 80 \%)$ e $9(>80 \%)$. Foram realizadas cinco avaliações, em intervalos de sete dias, a partir de oitenta dias após a emergência das plantas. Esses dados foram utilizados para calcular a área abaixo da curva de progresso da doença (AACPD), conforme Shanner \& Finney (1977), ou seja, a AACPD foi calculada com base no valor de severidade correspondente ao ponto médio de cada nota.

Os dados daAACPD foram submetidos, inicialmente, à análise de variância individual para cada local. A princípio, foram realizados os testes de aditividade dos efeitos do modelo e a normalidade dos erros. Não havendo nenhuma restrição a essas pressuposições, foram realizadas as análises de variâncias individuais. De posse dos resultados dessas análises, também foi verificada a pressuposição de homogeneidade de variâncias pelo teste de Hartley, citado por Gomes (1990), o qual se baseia na divisão do maior quadrado médio do resíduo pelo menor quadrado médio do resíduo das análises individuais. Sendo o valor resultante inferior a sete, infere-se homogeneidade de variâncias, o que possibilitou a análise conjunta dos experimentos. Assim, os dados de cada variável, obtidos em cada experimento e em cada local, foram submetidos a uma análise de variância conjunta, envolvendo os experimentos conduzidos nos três locais.

Para as estimativas dos parâmetros de estabilidade, foi utilizada a metodologia proposta por Eberhart \& Russell (1966). Para isso, as diferentes épocas de avaliação das doenças foram consideradas como ambientes diferentes e as análises foram realizadas separadamente para cada experimento. Utilizou-se o seguinte modelo de regressão:

$\mathrm{Y}_{\mathrm{ij}}=\mathrm{m}+\beta_{\mathrm{i}} \mathrm{I}_{\mathrm{j}}+\delta_{\mathrm{ij}}+\mathrm{e}_{\mathrm{ij}}$

em que

$\mathrm{Y}_{\mathrm{ij}}$ - severidade de doença observada para o híbrido i na época de avaliação j;

$\mathrm{m}$ - média do híbrido i em todas as épocas de avaliação;

$\beta_{\mathrm{i}}$ - coeficiente de regressão que mede a resposta do hibrido i às variações das épocas de avaliação;

$I_{j}$ - índice ambiental, obtido pela diferença entre a média

da época de avaliação $\mathrm{j}$ e a média de todas as épocas de avaliação j;

$\delta_{\mathrm{ij}}$ - desvio de regressão do híbrido i na época de avaliação $\mathrm{j}$;

$\mathrm{e}_{\mathrm{ij}}$ - erro experimental médio associado à média do material genético i na época de avaliação j.

Foram obtidas, também, estimativas de correlação classificatória de Spearman (Stell \& Torrie, 1980), entre as estimativas da AACPD dos híbridos nos diferentes experimentos, considerando cada doença separadamente e também entre a AACPD e a severidade média de doença dos híbridos nas diferentes épocas de avaliação (notas) e o coeficiente de regressão linear $\left(b_{1}\right)$ obtido na análise de estabilidade. As análises foram feitas nos programas Statistical Analysis System (SAS Institute Inc., 1993) e Sisvar (Ferreira, 2000). As médias dos híbridos foram agrupadas pelo teste de Scott-Knott $(\mathrm{p} \leq 0,05)$.

\section{RESULTADOS E DISCUSSÃO}

$\mathrm{Na}$ análise de variância conjunta para os dados da AACPD, envolvendo os três locais e as duas doenças avaliadas, houve diferenças significativas $(p \leq 0,01)$ para as fontes de variação híbridos, locais e interação híbridos x locais, para ambas as doenças, ou seja, ocorreu variabilidade entre os doze híbridos para a reação às doenças. A precisão experimental, avaliada pelo coeficiente de variação, foi de 36,97 e 48,23, para Cercosporiose e Mancha Branca, respectivamente. A coincidência no comportamento dos híbridos nos diferentes experimentos, avaliada pelo coeficiente de correlação de Spearman, foi alta e significativa, para todas as combinações possíveis entre os experimentos, exceto para Mancha-Branca, em uma combinação, em que o valor obtido foi baixo e não significativo (Tabela 2). Isso pode ser explicado pela menor severidade de Mancha Branca em alguns experimentos, o que dificultou a discriminação dos híbridos, reduzindo as estimativas das correlações obtidas.

Menores estimativas da correlação classificatória de Spearman seriam esperadas se as raças dos patógenos variassem entre os locais. No caso da Cercosporiose, esta é representada por dois grupos distintos (I e II) (Dunkle \& Levy, 2000; Carson et al., 2002; Brunelli, 2004). O grupo II caracteriza-se por apresentar taxa de crescimento mais lenta e por não produzir cercosporina em meio de cultura, quando comparada ao grupo I (Dunkle \& Levy, 2000; Brunelli, 2004). Existe, ainda, predominância de um grupo sobre o outro, de acordo com a geografia considerada. O grupo I prevalece em várias regiões do Brasil, enquanto o grupo II parece ser predominante somente no estado de Goiás (Brunelli, 2004). A ocorrência de grupos distintos em

TABELA 2 - Coeficientes de correlação de Spearman entre os diferentes experimentos para a área abaixo da curva de progresso de doença, considerando 12 híbridos avaliados em três locais

\begin{tabular}{lcc}
\hline \hline & \multicolumn{2}{c}{ AACPD } \\
\cline { 2 - 3 } & Cercosporiose & Mancha-Branca \\
\hline Lavras x Patos de Minas & $0,81^{* *}$ & $0,76^{* *}$ \\
Lavras x Passos & $0,94^{* *}$ & $0,58^{*}$ \\
Patos de Minas x Passos & $0,81^{* *}$ & $0,45^{\mathrm{ns}}$ \\
\hline
\end{tabular}

* e ** Significativo, a $5 \%$ e a $1 \%$ de probabilidade, respectivamente $\left(\mathrm{H}_{0}: \mathrm{r}=0\right)$. 
diferentes locais pode ser um indicativo da variabilidade da resistência dos genótipos avaliados, porém, os valores significativos da correlação para Cercosporiose indicam a hipótese de uma mesma raça do patógeno. Outro aspecto importante, que também sugere a especialização deste patógeno, é observar, em várias regiões do Brasil, ao longo dos anos, a redução no nível de resistência dos híbridos é uma conseqüência de possíveis alterações na população do patógeno por pressão de seleção direcional em favor de raças mais agressivas ou virulentas, acelerada por aumento no potencial de inóculo em função de plantio direto associado ao monocultivo, ausência de rotação e o uso de fungicidas.

$\mathrm{Na}$ avaliação das doenças nos diferentes ambientes, constataram-se altas severidades como indicado pelos valores de AACPD de Cercosporiose apenas em Passos $(498,83)$ e em Patos de Minas $(662,18)$, enquanto em Lavras ela foi considerada baixa $(203,29)$ (Tabela 3$)$. A baixa severidade da doença em Lavras, provavelmente, está ligada às baixas precipitações ocorridas na época de avaliação da doença (95 DAE), impedindo o seu progresso. Longos períodos de umidade relativa do ar próxima a $100 \%$, com ausência de água livre sobre a folha, são importantes para o início da infecção (Ringer \& Grybauskas, 1995). Com relação à Mancha Branca, constatou-se alta severidade da doença, indicado pelo valor da AACPD, em Lavras $(568,41)$ e em Patos de Minas $(591,99)$, enquanto em Passos praticamente não foi observada a doença, com média de 74,33 (Tabela 3).

A severidade das doenças registrada em todos os experimentos foi suficiente para discriminar os híbridos quanto à sua resistência (Tabela 4). Independentemente do local de avaliação, foram observadas diferenças entre os híbridos avaliados. Considerando os dados da AACPD em Lavras, observou-se que os híbridos mais suscetíveis à Cercosporiose foram P30F44, P30F53 e AG8021, enquanto os outros apresentaram comportamento semelhante, com baixa severidade da doença. Com relação à Mancha Branca, os híbridos mais suscetíveis foram P30F53 e P30K64, enquanto os híbridos mais resistentes foram AG7010, AG7088, Impacto, 2B707 e DKB390 (Tabela 4).

TABELA 3 - Valores de área abaixo da curva de progresso da doença (AACPD) obtidos a partir de avaliações da severidade da Cercosporiose e da Mancha-Branca, a partir de 12 híbridos comerciais de milho em três locais de avaliação

\begin{tabular}{lcc}
\hline \hline \multirow{2}{*}{ Local } & \multicolumn{2}{c}{ Doenças } \\
\cline { 2 - 3 } & Cercosporiose & Mancha-Branca \\
\hline Lavras & $203,29 \mathrm{a}$ & $568,41 \mathrm{~b}$ \\
Passos & $498,83 \mathrm{~b}$ & $74,33 \mathrm{a}$ \\
Patos de Minas & $662,18 \mathrm{c}$ & $591,99 \mathrm{~b}$ \\
MÉDIA & 454,77 & 411,58 \\
\hline
\end{tabular}

Médias seguidas de mesmas letras na coluna pertencem ao mesmo agrupamento, pelo teste de Scott Knott, a 5\% de significância.
Observou-se que houve baixa severidade de ManchaBranca em Passos, como pode ser verificado pelos valores de AACPD obtidos neste local, não tendo sido possível detectar diferenças significativas entre os híbridos avaliados, enquanto que, para a Cercosporiose, observou alta severidade da doença, sendo que os híbridos mais suscetíveis foram, novamente, P30F44, P30F53 e AG8021, corroborando os dados de Lavras, onde estes híbridos também foram os mais suscetíveis. Os híbridos mais resistentes à Cercosporiose, em Passos, foram 2B587, 2B707, AG7010, AG7088, AG8088 e Impacto, apresentado baixas severidade da doença. Em Pato de Minas, observaram-se diferenças significativas entre os híbridos quanto ao nível de resistência a ambas as doenças avaliadas. Com relação à Mancha-Branca, os híbridos mais suscetíveis foram P30F44, P30F53, P30K64, AG8021 e Impacto. Para os híbridos mais resistentes não foram observadas diferenças significativas, com destaque para AG7010 e AG7088 (Tabela 4).

As reações apresentadas pelos híbridos P30K64, DKB177, DKB390 e Impacto foi variável com o local de avaliação. O híbrido Impacto, por exemplo, apresentou boa resistência às doenças avaliadas em Lavras e em Passos, porém, foi suscetível, à Cercosporiose e à ManchaBranca, em Patos de Minas. Tal variação na severidade da doença, pode ter sido determinada pela instabilidade dos locos de resistência na sua interação com o ambiente ou por variações na população do patógeno nos diferentes ambientes de avaliação (Carson et al., 2002). No entanto, já foi constatado que híbridos resistentes a Cercosporiose que têm bom desempenho em uma área podem não apresentar desempenho satisfatório em outra (Dunkle \& Carson, 1998). A variação de resistência dos híbridos comerciais em diferentes regiões indica que o patógeno pode apresentar raças, cujo grau de virulência varia (Brunelli, 2004).

Outra alternativa para se avaliar o nível de resistência de híbridos é por meio de parâmetros de estabilidade fenotípica, como já empregado para outros patógenos, como as ferrugens do milho causadas por Puccinia polysora e Physopella zeae (Pinho et al., 2001). Para isso, é necessário proceder às análises de variância envolvendo as diferentes épocas de avaliação da severidade de doença. Constataramse, de modo geral, diferenças significativas $(\mathrm{p} \leq 0,01)$ para as fontes de variação dos híbridos, épocas de avaliação e interação híbrido $\mathrm{x}$ épocas de avaliação, em todos os experimentos e para ambos os patógenos. Nas Tabelas 5 e 6 são apresentadas as estimativas dos parâmetros de estabilidade pelo método de Eberhart \& Russel (1966).

Os híbridos AG7010, AG7088, 2B587 e 2B707 podem ser considerados os mais resistentes à Cercosporiose, em função das estimativas de $b_{0}$ e $b_{1}$ (Tabela 5). A pequena estimativa de $b_{0}$ obtida para esses híbridos, em todos os ambientes, indica a menor severidade da doença enquanto a pequena estimativa do coeficiente de regressão linear $\left(b_{1}\right)$ mostra o menor progresso da doença nestes híbridos . No caso do híbrido Impacto, as estimativas de $b_{1}$ em pelo menos um local (Patos de Minas) foram acima da média, não 
Reação de híbridos de milho e comparação de métodos para avaliação...

TABELA 4 - Valores de área abaixo da curva de progresso da doença (AACPD) de 12 híbridos comerciais de milho obtidos a partir de avaliações da severidade da Mancha-Branca (MB) e Cercosporiose (CE), em três locais de avaliação

\begin{tabular}{|c|c|c|c|c|c|c|}
\hline \multirow[t]{2}{*}{ Híbridos } & \multicolumn{2}{|c|}{ Lavras } & \multicolumn{2}{|c|}{ Passos } & \multicolumn{2}{|c|}{ Patos de Minas } \\
\hline & MB & $\mathrm{CE}$ & MB & $\mathbf{C E}$ & MB & $\mathbf{C E}$ \\
\hline 2B587 & $340,67 \mathrm{~b}$ & $6,42 \mathrm{a}$ & $28,67 \mathrm{a}$ & $42,33 \mathrm{a}$ & $3,50 \mathrm{a}$ & $228,08 \mathrm{a}$ \\
\hline 2B707 & $112,00 \mathrm{a}$ & $37,33 \mathrm{a}$ & $39,33 \mathrm{a}$ & $64,33 \mathrm{a}$ & $153,41 \mathrm{a}$ & $344,17 \mathrm{a}$ \\
\hline P30F44 & $1551,67 \mathrm{~d}$ & $736,75 \mathrm{c}$ & $135,33 \mathrm{a}$ & $1516,67 \mathrm{c}$ & $992,25 \mathrm{~b}$ & $2076,67 \mathrm{c}$ \\
\hline P30F53 & $1226,75 \mathrm{c}$ & $783,42 \mathrm{c}$ & $103,67 \mathrm{a}$ & $1307,33 \mathrm{c}$ & $1656,67 \mathrm{~b}$ & $1703,33 \mathrm{~b}$ \\
\hline P30K64 & $1341,67 \mathrm{~d}$ & $272,42 \mathrm{a}$ & $156,67 \mathrm{a}$ & $363,00 \mathrm{~b}$ & $1237,25 \mathrm{~b}$ & $169,75 \mathrm{a}$ \\
\hline AG 7010 & $0,00 \mathrm{a}$ & $0,00 \mathrm{a}$ & $63,67 \mathrm{a}$ & $129,67 \mathrm{a}$ & $0,00 \mathrm{a}$ & $0,58 \mathrm{a}$ \\
\hline AG 7088 & $0,00 \mathrm{a}$ & $0,00 \mathrm{a}$ & $3,33 \mathrm{a}$ & $14,00 \mathrm{a}$ & $0,00 \mathrm{a}$ & $0,58 \mathrm{a}$ \\
\hline AG 8021 & $1047,08 \mathrm{c}$ & $464,91 b$ & $47,33 \mathrm{a}$ & $1353,33 \mathrm{c}$ & $1178,92 \mathrm{~b}$ & $1435,00 \mathrm{~b}$ \\
\hline AG 8088 & $469,00 \mathrm{~b}$ & $13,42 \mathrm{a}$ & $62,00 \mathrm{a}$ & $159,00 \mathrm{a}$ & $251,42 \mathrm{a}$ & $191,33 \mathrm{a}$ \\
\hline DKB 177 & $481,25 \mathrm{~b}$ & $37,33 \mathrm{a}$ & $211,33 \mathrm{a}$ & $329,67 \mathrm{~b}$ & $457,92 \mathrm{a}$ & $145,25 \mathrm{a}$ \\
\hline DKB 390 & $225,16 \mathrm{a}$ & $86,33 \mathrm{a}$ & $0,00 \mathrm{a}$ & $575,67 \mathrm{~b}$ & $86,92 \mathrm{a}$ & $204,17 \mathrm{a}$ \\
\hline Impacto & $25,67 \mathrm{a}$ & $1,17 \mathrm{a}$ & $40,67 \mathrm{a}$ & $131,00 \mathrm{a}$ & $1085,58 \mathrm{~b}$ & $1447,25 \mathrm{~b}$ \\
\hline MÉDIA & 568,41 & 203,29 & 74,33 & 498,83 & 591,98 & 662,18 \\
\hline
\end{tabular}

Médias seguidas de mesmas letras na coluna pertencem ao mesmo agrupamento, pelo teste de Scott Knott, a 5\% de significância.

TABELA 5 - Severidade de Cercosporiose $\left(b_{0}\right)$, coeficientes de regressão $\left(b_{1}\right)$ e coeficientes de determinação $\left(\mathrm{R}^{2}\right)$ de 12 híbridos comerciais em três locais de avaliação

\begin{tabular}{|c|c|c|c|c|c|c|c|c|c|c|c|c|}
\hline \multirow[t]{2}{*}{ Híbridos } & \multicolumn{4}{|c|}{ Lavras } & \multicolumn{4}{|c|}{ Passos } & \multicolumn{4}{|c|}{ Patos de Minas } \\
\hline & $\mathbf{b}_{0}$ & $b_{1}$ & $\operatorname{Pr}>|t|^{* * *}$ & $\mathbf{R}^{2}$ & $\mathbf{b}_{0}$ & $\mathbf{b}_{1}$ & $\operatorname{Pr}>|t|$ & $\mathbf{R}^{2}$ & $\mathbf{b}_{0}$ & $\mathbf{b}_{1}$ & $\operatorname{Pr}>|t|$ & $\mathbf{R}^{2}$ \\
\hline 2B587 & 1,40 & 0,75 & 0,25 & 79,17 & 1,60 & 0,47 & 0,01 & 79,04 & 2,40 & 0,88 & 0,58 & 93,86 \\
\hline 2B707 & 1,40 & 0,75 & 0,25 & 79,17 & 1,80 & 0,79 & 0,29 & 92,84 & 2,80 & 0,76 & 0,26 & 80,63 \\
\hline P30F44 & 3,80 & 2,19 & 0,00 & 92,11 & 5,00 & 1,54 & 0,01 & 99,28 & 6,60 & 1,69 & 0,00 & 97,47 \\
\hline P30F53 & 4,00 & 2,36 & 0,00 & 94,74 & 5,00 & 1,54 & 0,01 & 99,28 & 5,80 & 1,95 & 0,00 & 97,96 \\
\hline P30K64 & 3,00 & 0,98 & 0,95 & 82,24 & 2,80 & 0,79 & 0,29 & 92,84 & 2,00 & 0,94 & 0,77 & 84,23 \\
\hline AG 7010 & 1,00 & 0,00 & 0,00 & 0,00 & 1,00 & 0,00 & 0,00 & 0,00 & 1,00 & 0,00 & 0,00 & 0,00 \\
\hline AG 7088 & 1,00 & 0,00 & 0,00 & 0,00 & 1,20 & 0,31 & 0,00 & 50,94 & 1,00 & 0,00 & 0,00 & 0,00 \\
\hline AG 8021 & 3,40 & 1,73 & 0,00 & 97,98 & 5,00 & 1,54 & 0,01 & 99,28 & 4,80 & 1,77 & 0,00 & 93,86 \\
\hline AG 8088 & 1,20 & 0,57 & 0,05 & 69,16 & 2,20 & 0,75 & 0,21 & 84,55 & 2,20 & 0,80 & 0,34 & 88,13 \\
\hline DKB 177 & 1,40 & 0,75 & 0,25 & 79,17 & 2,60 & 1,42 & 0,03 & 91,23 & 2,00 & 0,63 & 0,08 & 76,62 \\
\hline DKB 390 & 1,60 & 1,32 & 0,14 & 92,29 & 3,20 & 1,86 & 0,00 & 97,02 & 2,60 & 1,14 & 0,48 & 96,22 \\
\hline Impacto & 1,20 & 0,57 & 0,05 & 69,16 & 2,00 & 0,94 & 0,78 & 92,47 & 5,00 & 1,39 & 0,05 & 93,29 \\
\hline $\mathbf{r}\left(\mathbf{b}_{0} \mathbf{x} \mathbf{b}_{1}\right)^{*}$ & \multicolumn{4}{|c|}{0,91} & \multicolumn{4}{|c|}{0,83} & \multicolumn{4}{|c|}{0,92} \\
\hline
\end{tabular}

${ }^{*}$ Coeficiente de correlação

** Nível de significância

permitindo inferir que ele seja resistente à Cercosporiose em todos os ambientes.

Por outro lado, como materiais mais suscetíveis estão os híbridos P30F44 e P30F53, que apresentaram as maiores severidades médias da doença e com progresso da doença acima da média obtida em cada época de avaliação, com isso, esses híbridos apresentaram maiores estimativas, em todos os ambientes, tanto de $b_{0}$ como de $b_{1}$. Ocorreu associação positiva e alta entre as estimativas de $b_{0}$ e $b_{1}$, para todos os ambientes (Tabela 5). Essa é uma situação favorável, pois se podem utilizar tanto as estimativas de $b_{0}$ como de $b_{1}$ na discriminação dos híbridos. As estimativas de $b_{1}$ possibilitam inferir sobre a taxa de progresso da doença. Assim, $b_{1}$ superior a 1,0 indica que, para aquele híbrido, o progresso da doença foi acima da média e, portanto, ele é mais suscetível.
Nos casos dos híbridos mais suscetíveis à Cercosporiose, os valores de $\mathrm{R}^{2}$ foram os mais altos (Tabela 5). Em princípio, isso indica um melhor ajuste dos dados à equação de regressão proposta. Entretanto, para os híbridos mais resistentes, constatou-se que os valores de $\mathrm{R}^{2}$ foram os mais baixos, indicando um pior ajuste dos dados à equação de regressão. Contudo, deve-se enfatizar que, nesses casos, como as notas foram sempre baixas, pequenas flutuações acarretam proporcionalmente maiores desvios no modelo e, em consequência, menores valores de $\mathrm{R}^{2}$.

As estimativas dos parâmetros de estabilidade para a Mancha-Branca são apresentadas na Tabela 6. O híbrido AG7010 mostrou ser um dos mais resistentes, como já havia sido verificado através dos dados de AACPD. No caso do híbrido AG7088, foi detectada uma estimativa de 
A.H. Brito et al.

TABELA 6 - Severidade de Mancha Branca $\left(b_{0}\right)$, coeficientes de regressão $\left(b_{1}\right)$ e coeficientes de determinação $\left(\mathrm{R}^{2}\right)$ de 12 híbridos em três locais de avaliação

\begin{tabular}{|c|c|c|c|c|c|c|c|c|c|c|c|c|}
\hline \multirow[t]{2}{*}{ Híbridos } & \multicolumn{4}{|c|}{ Lavras } & \multicolumn{4}{|c|}{ Passos } & \multicolumn{4}{|c|}{ Patos de Minas } \\
\hline & $\mathbf{b}_{0}$ & $b_{1}$ & $\operatorname{Pr}>\left.|t|\right|^{* *}$ & $\mathbf{R}^{2}$ & $\mathbf{b}_{0}$ & $\mathbf{b}_{1}$ & $\operatorname{Pr}>|\mathbf{t}|$ & $\mathbf{R}^{2}$ & $\mathbf{b}_{0}$ & $\mathbf{b}_{1}$ & $\operatorname{Pr}>|\mathbf{t}|$ & $\mathbf{R}^{2}$ \\
\hline 2B587 & 2,80 & 1,12 & 0,22 & 99,44 & 1,60 & 1,71 & 0,10 & 80,36 & 1,20 & 0,33 & 0,03 & 31,15 \\
\hline 2B707 & 2,00 & 0,50 & 0,00 & 88,09 & 1,60 & 1,04 & 0,91 & 80,03 & 1,80 & 1,01 & 0,97 & 78,88 \\
\hline P30F44 & 5,40 & 1,44 & 0,00 & 95,89 & 2,40 & 1,04 & 0,91 & 80,03 & 4,40 & 1,18 & 0,56 & 94,15 \\
\hline P30F53 & 4,60 & 1,74 & 0,00 & 99,33 & 2,20 & 0,66 & 0,44 & 48,61 & 5,60 & 1,63 & 0,04 & 80,34 \\
\hline P30K64 & 5,00 & 1,41 & 0,00 & 99,34 & 1,80 & 1,71 & 0,10 & 91,84 & 4,40 & 2,03 & 0,00 & 96,76 \\
\hline AG 7010 & 1,00 & 0,00 & 0,00 & 0,00 & 1,80 & 0,57 & 0,33 & 35,71 & 1,00 & 0,00 & 0,00 & 0,00 \\
\hline AG 7088 & 1,00 & 0,00 & 0,00 & 0,00 & 1,40 & 1,04 & 0,91 & 80,03 & 1,00 & 0,00 & 0,00 & 0,00 \\
\hline AG 8021 & 4,40 & 1,44 & 0,00 & 95,89 & 2,00 & 0,00 & 0,02 & 0,00 & 4,40 & 1,18 & 0,56 & 94,15 \\
\hline AG 8088 & 3,00 & 1,41 & 0,00 & 99,34 & 1,60 & 1,04 & 0,91 & 80,03 & 2,40 & 1,18 & 0,56 & 94,15 \\
\hline DKB 177 & 3,00 & 1,41 & 0,00 & 99,34 & 2,00 & 2,09 & 0,01 & 96,03 & 2,80 & 1,74 & 0,02 & 96,67 \\
\hline DKB 390 & 2,20 & 1,20 & 0,04 & 93,22 & 1,00 & 0,00 & 0,02 & 0,00 & 1,80 & 0,50 & 0,11 & 70,08 \\
\hline Impacto & 1,20 & 0,29 & 0,00 & 74,33 & 1,60 & 1,04 & 0,91 & 80,03 & 4,40 & 1,18 & 0,56 & 94,15 \\
\hline
\end{tabular}

*Coeficiente de correlação

${ }^{* *}$ Nível de significância

$\mathrm{b}_{1}$ acima da média em, pelo menos, um local (Passos), não permitindo inferir que ele seja resistente ao patógeno em todos os ambientes. Os híbridos P30F44, P30K64, P30F53 e DKB177 mostraram maiores estimativas de $\mathrm{b}_{0}$ em todos os locais e $b_{1}$ acima de 1,0 , na maioria dos locais e, portanto, podem ser considerados os mais suscetíveis, como já fora verificado através das estimativas da AACPD.

O comportamento dos híbridos não foi coincidente nas diferentes épocas de avaliação, em função da presença da interação híbridos x época de avaliação. Isso implica na necessidade de efetuar mais de uma avaliação das doenças, o que, de certa forma, pode complicar o trabalho dos melhoristas. Utilizando esses dados para a determinação da resistência dos materiais, as opções são as metodologias da AACPD ou as estimativas dos parâmetros de estabilidade.

As estimativas da correlação classificatória de Spearman (Tabela 7) quanto à resistência dos híbridos, obtida pelas duas metodologias, foram positivas e significativas em todos os locais, para ambas as doenças, indicando que, em se tratando desses patógenos, houve coincidência na avaliação do progresso das doenças pelas duas metodologias. No caso de ferrugem do milho cujo agente etiológico é o fungo Puccinia polysora, Pinho et al. (2001) observaram que tanto a área abaixo da curva de progresso da doença quanto os parâmetros da estabilidade fenotípica mostraram-se eficientes na discriminação do nível de resistência dos híbridos, permitindo a classificação de modo semelhante. $\mathrm{O}$ conhecimento da eficiência das metodologias de avaliação, da área abaixo da curva de progresso da doença e dos parâmetros da estabilidade fenotípica é de extrema importância, visto que alguns milhares de híbridos e/ou linhagens deverão ser avaliados para a obtenção de materiais resistentes às doenças já citadas.
TABELA 7 - Coeficientes de correlação de Spearman entre a área abaixo da curva de progresso da doença (AACPD) e a severidade média de doença $\left(b_{0}\right)$ e os coeficientes de regressão $\left(b_{1}\right)$ obtidos a partir da avaliação da severidade da Cercosporiose e da Mancha Branca em 12 híbridos comerciais em três locais

\begin{tabular}{lclc}
\hline \hline & Lavras & Passos & Patos de Minas \\
\hline Cercosporiose & & & \\
AACPD x b $b_{0}$ & $0,97 * *$ & $0,97^{* *}$ & $0,98^{* *}$ \\
AACPD x b & $0,92^{* *}$ & $0,73^{* *}$ & $0,86^{* *}$ \\
& & & \\
Mancha-Branca & & & \\
AACPD x b & $0,98^{* *}$ & $0,69 *$ & $0,99^{* *}$ \\
AACPD x $\mathrm{b}_{1}$ & $0,78^{* *}$ & $0,59^{*}$ & $0,75^{* *}$ \\
\hline
\end{tabular}

** - Significativamente diferente de zero, a $1 \%$ de probabilidade.

\section{AGRADECIMENTOS}

À Fundação de Amparo a Pesquisa do Estado de Minas Gerais - FAPEMIG pelo apoio financeiro (Proc. CAG 621/06).

\section{REFERÊNCIAS BIBLIOGRÁFICAS}

Brito AH, Pinho RG von, Pozza EA, Pereira JLAR, Faria Filho EM (2007) Efeito da cercosporiose no rendimento de híbridos comerciais de milho. Fitopatologia Brasileira 32:472-479.

Bruneli KR (2004) Cercospora zea-maydis: esporulação, diversidade morfo-genética e reação de linhagens de milho. Tese de Doutorado. ESALQ - Universidade de São Paulo. Piracicaba SP.

Carson ML, Goodman MM, Williamson SM (2002) Variation in aggressiveness among isolates of Cercospora from maize as a

Tropical Plant Pathology 36 (1) January - February 2011 
Reação de híbridos de milho e comparação de métodos para avaliação...

potential cause of genotype-environment interaction in gray leaf spot trials. Plant Disease 86:1089-1093.

Dunkle LD, Carson ML (1998) Genetic variation in Cercospora and the potential impact on selecting for resistance to gray leaf spot of corn. In: Annual Corn and Sorghum Research Conference. Chicago. American Seed Trade Association. pp. 30-35.

Dunkle LD, Levy M. (2000) Genetic relatedness of African and United States populations of Cercospora zea-maydis. Phytopathology 90:486-490.

Eberhart SA, Russell WA (1966) Stability parameters for comparing varieties. Crop Science 6:36-40.

Fernandes FT, Oliveira E (1997) Principais doenças da cultura do milho. 26th Ed. Sete Lagoas MG. Embrapa Milho e Sorgo.

Ferreira DF (2000) Sisvar: sistema de análise de variância. Versão 3.04 Lavras MG.

Gomes FP (1990) Curso de estatística experimental. Piracicaba SP. Editora Nobel.

Juliatti FC, Appelt CCNS, Brito $\mathrm{CH}$, Gomes LS, Brandão AM, Hamawaki OT, Melo B (2004) Controle da feosféria, ferrugem comum e cercosporiose pelo uso da resistência genética, fungicidas e épocas de aplicação na cultura do milho. Bioscience Journal 20:45-54.

Paccola-Meirelles LD, Ferreira AS, Meirelles WF, Marriel IE, Casela CR (2001) Detection of a bacterium associated with a leaf spot disease of maize in Brazil. Journal of Phytopathology 49:275279.

Pereira DG, Sediyama T, Cruz CD, Gomes JL, Teixeira RC (1996) Análise da estabilidade fenotípica de cultivares de soja (Glycine $\max ($ L.) Merril) em relação a diferentes isolados de Diaporthe phaseolorum f. sp. meridionalis. Revista Ceres 43:743-754.

Pinho RG von, Ramalho MAP, Resende IC, Silva HP, Pozar G (2001) Reação de híbridos comerciais de milho às ferrugens polissora e tropical. Pesquisa Agropecuária Brasileira 36:439445 .

Pozar G, Butruille D, Diniz HS, Viglioni JP (2009) Mapping and validation of quantitative trait loci for resistance to Cercospora infection in tropical maize (Zea mays L.). Theoretical and Applied Genetics 118:553-564.

Ringer CE, Grybauskas AP (1995) Infection cycle components and disease progress of gray leaf spot on field corn. Plant Disease 79:24-28.

SAS INSTITUTE INC (1993). SAS System. Cary, 18 v.

Shaner G, Finney RE (1977) The effect of nitrogen fertilization on the expression of slow-mildewing resistance in knox wheat. Phytopathology 70:1183-1186.

Stell RG, Torrie JK (1980) Principles and procedures of statistics: a biometrical approach. 2nd Ed. Tokyo. McGraw-Hill.

TPP 129 - Recebido 10 Junho 2010 - Aceito 17 Janeiro 2011 Editor de Seção: Carlos R. Casela 